\title{
Therapeutic electrical stimulation of injured peripheral nerve tissue using implantable thin-film wireless nerve stimulators
}

\author{
Matthew R. MacEwan, PhD, ${ }^{1,2}$ Paul Gamble, MD, ${ }^{2}$ Manu Stephen, $M S,{ }^{2}$ and Wilson Z. Ray, MD $^{2}$ \\ ${ }^{1}$ Department of Biomedical Engineering, Washington University; and 2Department of Neurosurgery, Washington University \\ School of Medicine, St. Louis, Missouri
}

OBJECTIVE Electrical stimulation of peripheral nerve tissue has been shown to accelerate axonal regeneration. Yet existing methods of applying electrical stimulation to injured peripheral nerves have presented significant barriers to clinical translation. In this study, the authors examined the use of a novel implantable wireless nerve stimulator capable of simultaneously delivering therapeutic electrical stimulation of injured peripheral nerve tissue and providing postoperative serial assessment of functional recovery.

METHODS Flexible wireless stimulators were fabricated and implanted into Lewis rats. Thin-film implants were used to deliver brief electrical stimulation ( $1 \mathrm{hour}, 20 \mathrm{~Hz}$ ) to sciatic nerves after nerve crush or nerve transection-and-repair injuries.

RESULTS Electrical stimulation of injured nerves via implanted wireless stimulators significantly improved functional recovery. Brief electrical stimulation was observed to increase the rate of functional recovery after both nerve crush and nerve transection-and-repair injuries. Wireless stimulators successfully facilitated therapeutic stimulation of peripheral nerve tissue and serial assessment of nerve recovery.

CONCLUSIONS Implantable wireless stimulators can deliver therapeutic electrical stimulation to injured peripheral nerve tissue. Implantable wireless nerve stimulators might represent a novel means of facilitating therapeutic electrical stimulation in both intraoperative and postoperative settings.

https://thejns.org/doi/abs/10.3171/2017.8.JNS163020

KEY WORDS electrical stimulation; peripheral nerve; nerve injury; nerve regeneration; medical device; wireless implant; rat model

$\mathrm{P}$ ERIPHERAL nerve injuries represent one of the most common causes of sensorimotor deficit and lost productivity in adults. ${ }^{35}$ Peripheral nerve injuries result in the interruption of axonal continuity, neuronal cell death, and Wallerian degeneration of distal fibers, which terminally lead to the loss of motor, sensory, and autonomic function. ${ }^{34}$ This series of events, which begins with nerve injury and culminates in loss of sensorimotor function, is a major cause of disability worldwide. In the United States alone, more than 50,000 peripheral nerve injuries are recorded annually and result in health care expenditures of more than $\$ 7$ billion/year. ${ }^{12}$ Therefore, great interest has been placed in improving surgical and rehabilitative strategies for treating peripheral nerve injuries and improving functional recovery.
In cases of simple peripheral nerve defects, sufficient functional recovery can be attained through tension-free, end-to-end coaptation of residual nerve stumps. In contrast, clinical management of complex nerve defects typically requires advanced surgical techniques, including the use of interpositional autologous nerve grafts (autografts), decellularized allografts, and/or nerve guidance conduits. ${ }^{6,9}$, $13,14,20,21,24,26,32,39,44$ Although nerve grafting represents the gold standard for the treatment of complex peripheral nerve injuries, the use of nerve autografts continues to present significant clinical limitations. Functional recovery after proximal autografting is frequently poor despite the regenerative capacity of injured peripheral axons and the supportive microenvironment of autologous nerve tissue. $3,7,8,22,23,33,42,45$ Given the known barriers to surgical re-

ABBREVIATIONS EDL = extensor digitorum longus; $\mathrm{EMG}=$ electromyographic; $\mathrm{GM}=$ gluteus maximus; $\mathrm{GS}=$ gastrocnemius; $\mathrm{PL}=$ plantaris; $\mathrm{TA}=$ tibialis anterior. SUBMITTED December 1, 2016. ACCEPTED August 1, 2017. 
pair of critical $(>5-\mathrm{cm})$ nerve defects and the temporal limits on functional end-organ reinnervation, existing surgical interventions are limited to select patient populations and postinjury time frames. As a result, great interest has been placed in the development of therapeutic adjuncts that can enhance nerve regeneration and functional recovery after nerve injury and nerve repair procedures.

Brief electrical stimulation of injured peripheral nerve tissue represents a promising method for enhancing axonal outgrowth and augmenting peripheral nerve function in vivo. Previous studies found that direct current electrical stimulation enhanced and guided neurite extension and axonal outgrowth in a variety of cell culture models. ${ }^{10,27-29,31,36-38}$ Brief low-frequency electrical stimulation of injured peripheral nerve tissue similarly was found to enhance the rate of axonal regeneration and functional recovery in vivo in a number of animal models. ${ }^{2,11,17}$ One hour of low-frequency electrical stimulation applied to rat nerve was observed to accelerate axonal outgrowth and promote regeneration of all motor axons., ${ }^{2,17}$ Electrical stimulation also was observed to accelerate axonal regeneration in mice. ${ }^{1,11}$ Similar results were observed after application of brief electrical stimulation to injured human peripheral nerve tissue. ${ }^{1}$ Brief electrical stimulation of the median nerve in humans during routine carpal tunnel release surgery was observed to improve postoperative functional recovery. The results of these studies suggest that the therapeutic value of electrical stimulation might be applicable equally in animal and human models.

Existing methods for clinically applying therapeutic electrical stimulation to injured peripheral nerves unfortunately are limited. In the majority of published study reports, electrical stimulation of peripheral nerve tissue was delivered using a nerve hook or wire electrodes applied directly to the target nerve during open surgical repair of the injured nerve. ${ }^{2,4,18}$ In this setting, the application of electrical stimulation is limited to a brief intraoperative period during which the patient is fully anesthetized and the target nerve is surgically exposed. The application of electrical stimulation is limited further by the fact that the terminal nerve hook electrodes must be connected physically to an external power source. Direct application of electrical stimulation thereby precludes postoperative treatment and repeat dosing without the use of a painful and nonspecific transcutaneous approach. Altogether, these restrictions limit the timing and duration of therapeutic stimulation and significantly increase the length and complexity of nerve repair procedures. Increased duration of anesthesia and operative procedures also can increase the risk of anesthesia-related complications and increase procedural costs.

A previous study conducted by Gamble et al. ${ }^{15}$ found that wireless thin-film receivers offer a low-profile passive implant capable of facilitating repeat electrical stimulation to rodent peripheral nerves for diagnostic assessment of nerve function after injury. In that study, the thin-film wireless receivers were found to reliably deliver electrical impulses to interfaced nerves, recruit peripheral nerve tissue, and activate distal musculature for the purpose of serially tracking postoperative functional recovery. The effects of wireless electrical stimulation on the rate and efficacy of peripheral nerve regeneration and motor recovery unfortunately were not examined. We designed our study to provide direct and independent examinations of the ability of novel wireless implantable nerve stimulators to deliver therapeutic electrical stimulation to injured rodent sciatic nerves and accelerate postoperative functional recovery. We also examined the application of previously validated brief electrical stimulation paradigms via novel wireless implants immediately after nerve injury and repair as a therapeutic means of accelerating functional recovery after nerve crush and nerve transection injuries.

\section{Methods \\ Experimental Design}

Twenty-five adult male Lewis rats were distributed randomly among 5 groups (I-V), with 5 animals in each group. Rats in all the groups underwent surgical implantation of a wireless nerve stimulator. Group I animals served as the positive controls and underwent surgical exposure of the sciatic nerve with no injury and implantation of a stimulator. Groups II-V served as the experimental groups; group II and III rats received a crush injury and underwent stimulator implantation, and group IV and V rats underwent a transection and repair and then stimulator implantation. All animals in groups III (crush) and $\mathrm{V}$ (transection) received 1 hour of therapeutic electrical stimulation $(2.5 \mathrm{~V}, 20 \mathrm{~Hz})$ delivered immediately after surgery via the implanted wireless nerve stimulator. Furthermore, all animals underwent weekly assessment of functional recovery through use of the stimulator to collect electromyographic (EMG) signal recordings in distal musculature. At the terminal time point of 13 weeks, animals in all groups underwent terminal assessment of functional recovery and nerve regeneration as measured via evoked muscle force measurement. After this assessment, each animal was killed, and its distal musculature was harvested to collect muscle mass data. All animal procedures were performed in strict accordance to the guidelines of the Division of Comparative Medicine and the Animal Studies Committee at Washington University School of Medicine in St. Louis.

\section{Assembly of Implantable Wireless Nerve Stimulator}

Wireless nerve stimulators were designed and constructed by Red Rock Laboratories. The stimulators consisted of 3 components: 1) a receiver coil with a demodulating circuit, 2) microwire leads, and 3) a silicone nerve cuff. The receiver coil (Fig. 1) comprised a spiral antenna, and surface mount components were soldered onto a flexible printed circuit board (PCB) substrate to complete the demodulating circuitry. The receiver coils were tuned to a carrier frequency of $5 \mathrm{MHz}$ and demodulated incoming signals. Two microwire leads, insulated with polytetrafluoroethylene and composed of Pt/Ir (Medwire, Sigmund Cohn Corp.), were soldered onto contact points of the PCB substrate to provide an electrical connection between the sciatic nerve and the receiver coil (Fig. 2). Uninsulated ends of the microwire leads were integrated into silicone nerve cuffs (inner diameter $2 \mathrm{~mm}$, outer diameter $4 \mathrm{~mm}$ ) to ensure robust peripheral nerve contact. After construc- 


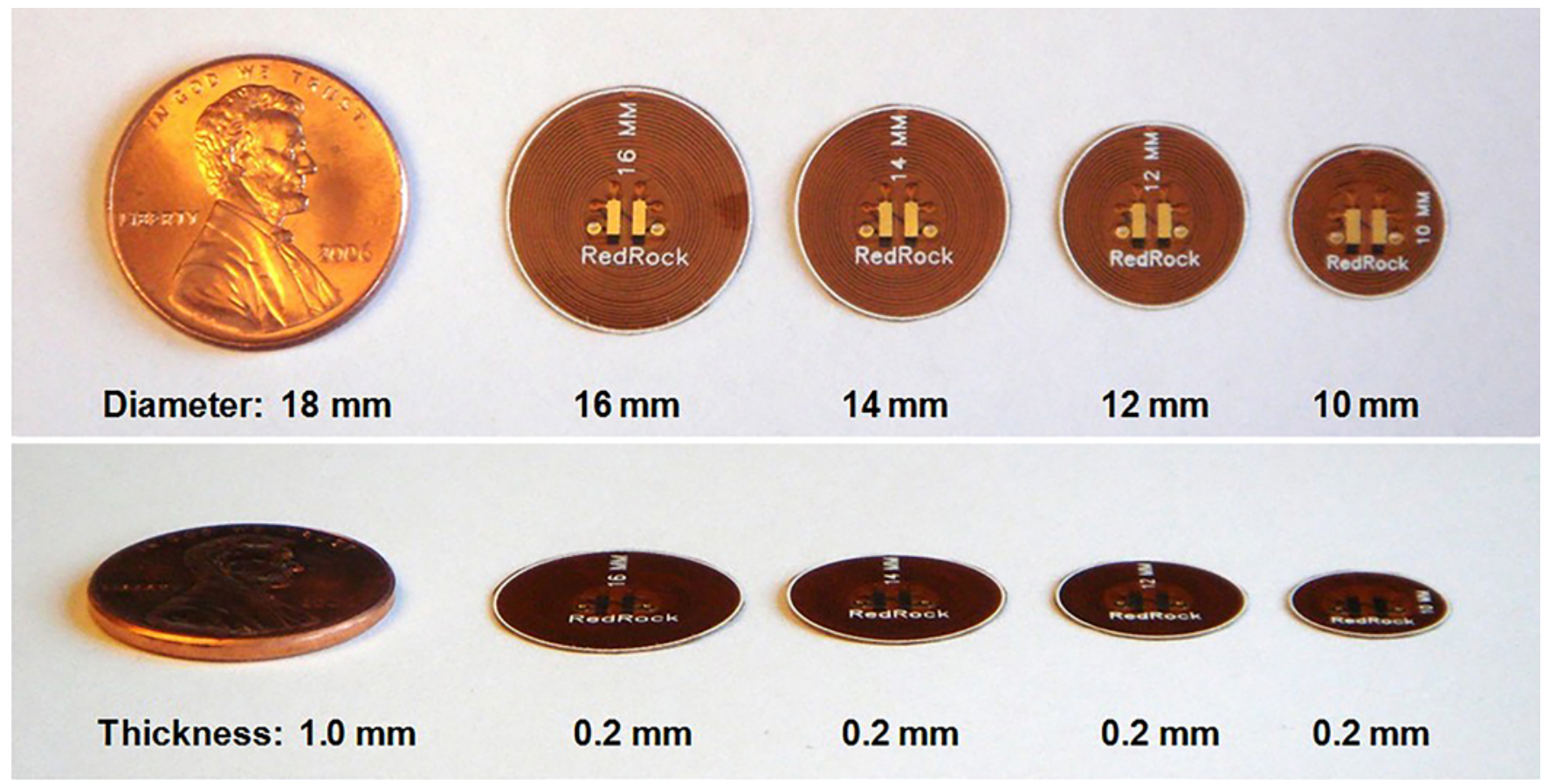

FIG. 1. Fabricated thin-film wireless receivers containing integrated spiral antennae, surface mount pads for fixation of tuning circuitry, and bonding sites for Pt/Ir leads. These thin-film receivers are a flexible, low-profile implant suitable for use in small rodent models. Figure is available in color online only.

tion, flexible receiver coils were potted in medical-grade silicone elastomer (type A) (Dow Corning). Completed wireless implants were sterilized with ethylene oxide gas before use in vivo. Modified class E oscillator circuits, tuned to the $5-\mathrm{MHz}$ carrier frequency, powered and activated the wireless nerve stimulators. Oscillator circuits were connected to circular transmitting coils and placed near the stimulators to provide inductive coupling and produce wireless power delivery to the implanted devices for peripheral nerve stimulation.

\section{Sciatic Nerve Injury}

Each animal was anesthetized with isoflurane (4\% at induction, 2\% throughout procedure) administered by in-

\section{Wireless Receiver}
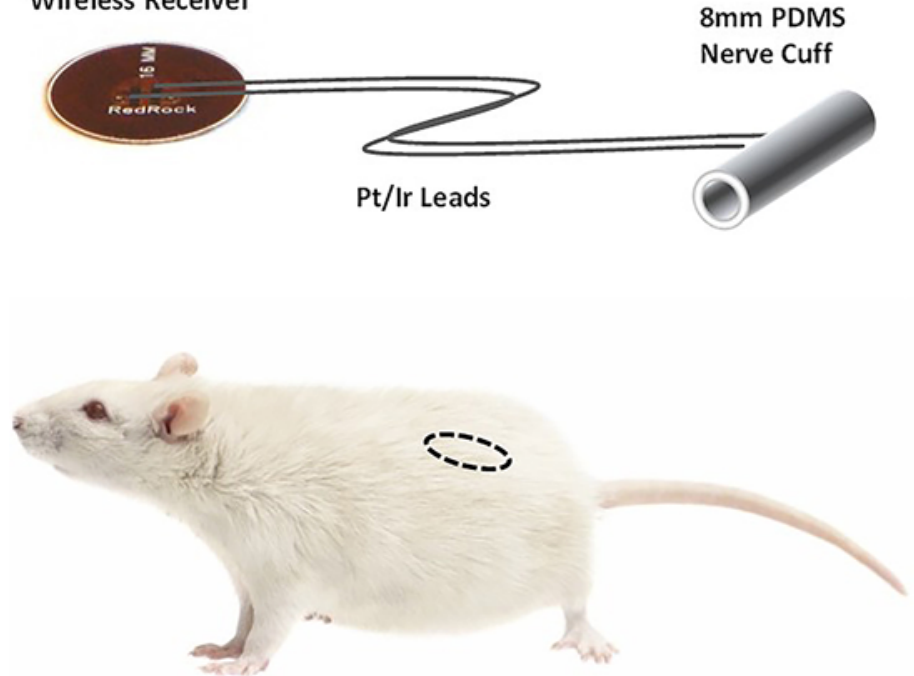

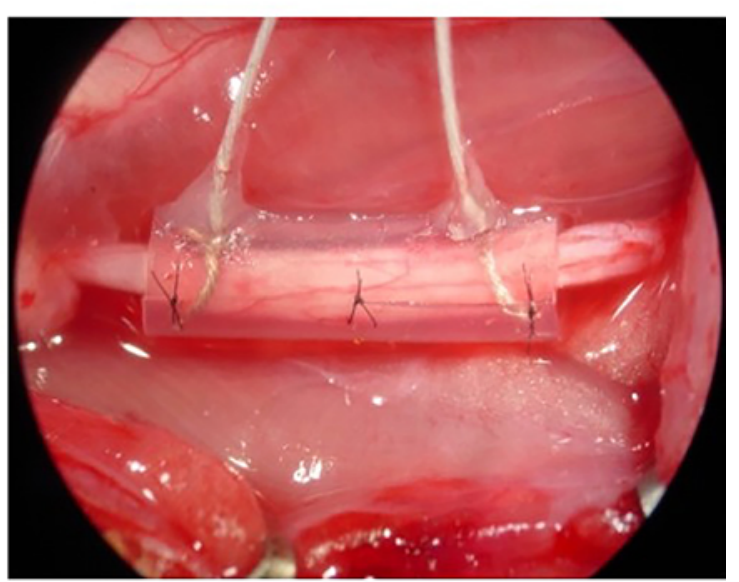

FIG. 2. Left: Assembly of thin-film wireless receivers for in vivo implantation. Flexible thin-film receivers are bonded to Pt/lr leads that are integrated into silicone nerve cuff electrodes sized for the rat sciatic nerve. Right: Implantable wireless nerve stimulators are implanted subcutaneously in laboratory rats and interfaced with the sciatic nerve. Shown is an intraoperative view of the implanted wireless nerve stimulator. Silicone nerve cuff electrodes wired to flexible thin-film receivers were applied to the rat sciatic nerve and secured using 8-0 nylon suture to facilitate an intimate noncompressive interface between active Pt/lr leads and peripheral nerve tissue. PDMS = polydimethylsiloxane. Figure is available in color online only. 
halation. The right sciatic nerve was exposed through a gluteal muscle-splitting incision. The animals in groups II and III received a reproducible crush injury $5 \mathrm{~mm}$ proximal to the sciatic nerve trifurcation using No. 5 Jeweler's forceps applied twice for 30 seconds each. Group IV and $\mathrm{V}$ rats underwent sciatic nerve transection and repair 5 $\mathrm{mm}$ proximal to the trifurcation. Nerves were transected with fine iris scissors and repaired via 10-0 nylon suture (Sharpoint, Surgical Specialties Corp.).

\section{Implantation of Wireless Nerve Stimulator}

Each animal underwent implantation of a wireless nerve stimulator (Fig. 2). A subcutaneous pocket was created on the back of the animal along the right leg to allow the wireless receiver to remain secured. The Pt/ Ir microwire leads were routed from the receiver to the exposed sciatic nerve. Silicone nerve cuffs were then secured around the sciatic nerve $5 \mathrm{~mm}$ proximal to the site of injury and closed via 10-0 nylon suture (Fig. 2). After implantation, the layer of muscle fascia was closed using 6-0 polyglactin (Vicryl, Ethicon), and the skin was closed with 4-0 nylon suture (Ethilon, Ethicon). The animals then were allowed to recover and monitored postoperatively before being returned to the animal housing facility.

\section{Therapeutic Electrical Stimulation}

After surgical implantation, wireless nerve stimulators in groups III and V delivered electrical stimulation to interfaced sciatic nerves to determine its therapeutic effects after nerve injury. Each animal was anesthetized with isoflurane as described previously, and a wireless transmitter coil was placed over the animal to activate the implanted wireless receiver. The implanted receivers delivered cathodic monophasic impulses (amplitude $2.5 \mathrm{~V}$, pulse duration $200 \mu \mathrm{sec}$ ) and were used to deliver brief electrical stimulation (duration 1 hour, amplitude $2.5 \mathrm{~V}$, frequency $20 \mathrm{~Hz}$ ) to injured sciatic nerves immediately after surgery in a manner consistent with that described for previous studies; wireless implants stimulated the injured sciatic nerve for a period of 1 hour at a frequency of 20 Hz. Preliminary studies conducted in the laboratory using healthy animals that had not undergone nerve repair and had uninjured sciatic nerve tissue validated the idea that electrical stimulation of the nerve under these parameters effectively and reliably recruits nerve fibers over the 1-hour period, as evidenced by muscle twitch and EMG measurements. After electrical stimulation, the animals were allowed to recover and returned to their enclosure.

\section{Assessment of Functional Recovery: Serial Wireless Nerve Stimulation}

Serial assessment of sciatic nerve recovery began 1 week after surgery. Electromyograms of distal musculature were recorded by observers blinded to group allocation. The animals were anesthetized as described previously, and a wireless transmitter coil was placed over each animal and centered over the implanted receiver to deliver cathodic monophasic electrical impulses to the sciatic nerve. The resulting electromyograms were recorded differentially through needle electrodes at muscles both proximal and distal to the implanted device, specifically, the gluteus maximus (GM), tibialis anterior (TA), gastrocnemius (GS), and plantaris (PL) muscles. Measured signals were band-pass filtered (low pass $1 \mathrm{~Hz}$, high pass 5 $\mathrm{kHz}$, notch $60 \mathrm{~Hz}$ ) and amplified by a factor of 1000 via a 2-channel microelectrode alternating current amplifier (model 1800, A-M Systems, Inc.). Filtered signals were then recorded onto a desktop PC equipped with custom data-acquisition software (Red Rock Laboratories). Baseline EMG measurements in healthy age-matched animals that had no nerve injury inflicted were performed similarly. EMG responses allowed for the mapping of stimulus amplitudes and frequencies to construct recruitment curves and determine peak-to-peak amplitudes. This serial assessment of EMG recordings and data acquisition was repeated weekly for 13 weeks after surgery, comparable to the process followed in previous rodent nerve injury studies.

\section{Assessment of Functional Recovery: Evoked Muscle Force Measurement}

At the terminal time point of 13 weeks after surgery, functional recovery of the sciatic nerve in all animals was determined by measuring force output in reinnervated musculature after stimulation of the nerve.16,25,30,41 Animals were anesthetized as previously described and underwent surgical exposure of the distal tendons of the extensor digitorum longus (EDL) and TA muscles. The exposed tendons were then secured onto a stainless steel $\mathrm{S}$ hook at the musculotendinous junction using 5-0 nylon suture. Animals were secured into a custom-built functional assessment station (FASt System, Red Rock Laboratories), and the right leg was immobilized at the femoral condyles to isolate force production. The stainless steel $\mathrm{S}$ hook was then connected to a 5-N thin-film load cell (S100, Strain Measurement Devices, Inc.) supported on an adjustable mount. Hook electrodes fabricated from silver wire (0.0055-inch coated silver) (A-M Systems) were connected to a stimulus isolator (model 2200, A-M Systems) and controlled by custom software (Red Rock Laboratories) to deliver cathodic monophasic impulses (duration $300 \mu \mathrm{sec}$, single-mode frequency $200 \mathrm{~Hz}$, amplitude 0.5 $\mathrm{mA}$ ) to the sciatic nerve proximal to the injury site. Resulting force production from the isolated EDL and TA muscles was transduced via the force sensor and recorded via the custom data-acquisition software.

\section{Statistical Analysis}

The results are reported as means \pm standard deviation, unless stated otherwise. Statistica 6.0 (StatSoft) was used to perform the statistical analyses. Specifically, data normality was confirmed via the Kolmogorov-Smirnov test $(\mathrm{p}>0.05)$. Comparisons between the experimental and control groups were performed using ANOVA with the Fisher least significant difference test with a statistical significance of $\mathrm{p}<0.05$.

\section{Results}

\section{Performance of Implantable Wireless Nerve Stimulators}

Benchtop testing was performed by approximating 
wireless transmitter coils and fabricated wireless nerve stimulators while measuring evoked stimulus output. Benchtop assessments revealed successful tuning and performance of all fabricated implantable wireless receivers. All receivers had stable output $(2.0-2.8 \mathrm{~V}$ at a $5.0-\mathrm{MHz}$ carrier frequency) while using a carrier signal with a peakto-peak voltage (Vpp) of 10 (Fig. 3).

The wireless nerve stimulators were implanted to evaluate the ability of the devices to deliver therapeutic electrical stimulation to target nerves and provide simultaneous serial assessment of postoperative functional recovery. Twenty-five thin-film wireless nerve stimulators were implanted into adult male Lewis rats and attached to the sciatic nerve via integrated nerve cuff electrodes. These implanted devices successfully delivered brief electrical stimulation to target nerves in all the animals, as evidenced by proximal muscle activation during treatment. The implanted devices also demonstrated stable and reliable recruitment of the interfaced peripheral nerve tissue during the course of the 13-week study in vivo, as evidenced by EMG recordings. All the implants were observed to remain operational up to the 13 -week terminal time point. Minimal fibrosis and encapsulation of the implanted device was noted in a majority of the rats.

\section{Postoperative Serial Assessments of Nerve and Muscle Function}

Serial activation of peripheral nerve tissue and innervated musculature via the implanted wireless stimulators was performed to track functional recovery after peripheral nerve injury in rats that were and in those that were not receiving therapeutic electrical stimulation. Wireless activation of all the nerve stimulators elicited activation of musculature both proximal (GM) and distal (TA, EDL, GS, and PL) to the site of nerve injury, as evidenced by recorded EMG activity (Figs. 4-7). Serial EMG measurements revealed various degrees of functional motor recovery over 13 weeks after injury and brief electrical stimulation.

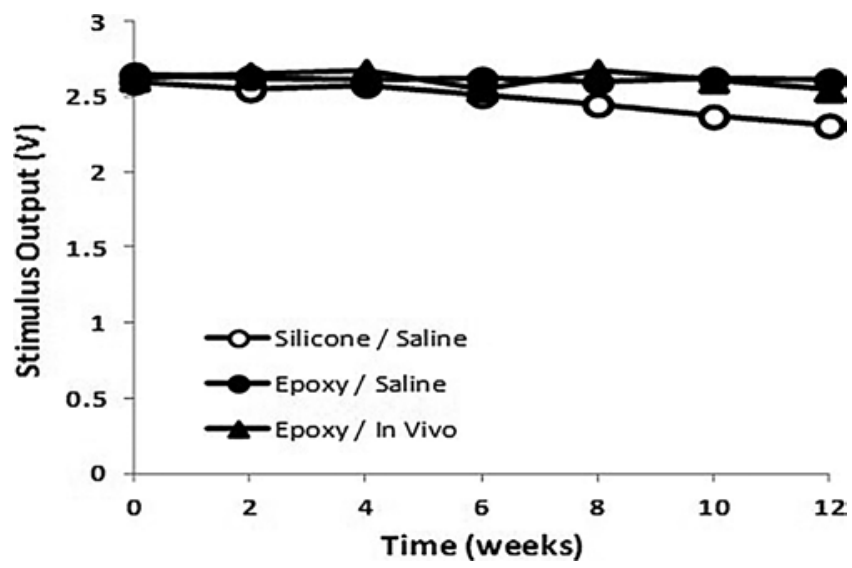

FIG. 3. Output voltage of potted wireless receivers after prolonged immersion in an aqueous bath of sterile saline. Submersion of potted receivers was performed to test the hermetic seal of implantable devices and confirm reliable repeatable activation of the constructed devices. Stable output of immersed receivers revealed reliable operation over prolonged periods of time in near-physiological conditions.

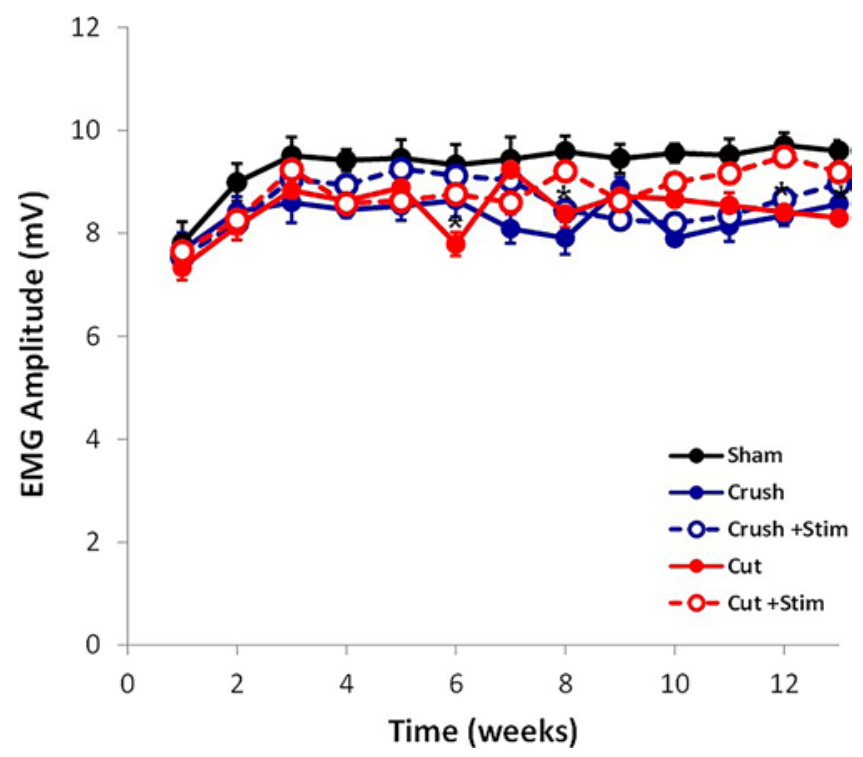

FIG. 4. Maximum amplitude of electromyograms evoked in the GM after electrical activation of uninjured, crushed, and cut-and-repaired sciatic nerves in both the presence and the absence of brief electrical stimulation via the implanted wireless nerve stimulator. Mean values and SDs are shown. * $p<0.05$ versus time-matched injury model with no electrical stimulation. Figure is available in color online only.

Evoked electromyograms recorded in the control animals (no nerve injury) revealed consistent amplitudes throughout the duration of the study (GM $9.60 \pm 0.24 \mathrm{mV}$, TA $10.31 \pm 0.47 \mathrm{mV}$, GS $10.15 \pm 0.45 \mathrm{mV}$, and PL 7.59 $\pm 0.38 \mathrm{mV}$ ). Consistent activation of musculature by the implanted wireless devices revealed the consistent nature of wireless nerve interfacing and the preservation of inter-

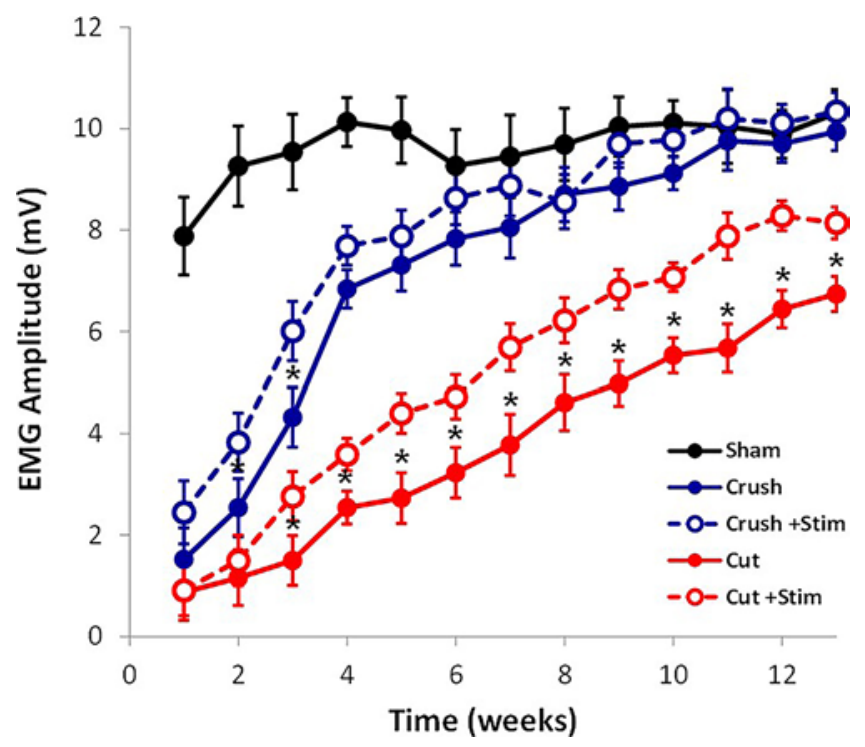

FIG. 5. Maximum amplitude of electromyograms evoked in the TA after electrical activation of uninjured, crushed, and cut-and-repaired sciatic nerves in both the presence and the absence of brief electrical stimulation via the implanted wireless nerve stimulator. Mean values and SDs are shown. ${ }^{*} p<0.05$ versus time-matched injury model with no electrical stimulation. Figure is available in color online only. 
faced peripheral nerve tissue and innervated musculature during long-term activation.

Directly after nerve crush and nerve transection injuries, evoked EMG responses revealed effective knockdown of activation in musculature distal to the site of nerve injury (TA/crush $1.52 \pm 0.69 \mathrm{mV}$, TA/cut $0.87 \pm 0.56 \mathrm{mV}$, GS/crush $2.38 \pm 0.65 \mathrm{mV}, \mathrm{GS} /$ cut $0.73 \pm 0.49 \mathrm{mV}, \mathrm{PL} /$ crush $1.25 \pm 0.62 \mathrm{mV}$, and PL/cut $0.87 \pm 0.47 \mathrm{mV}$ ). Evoked EMG responses in musculature proximal to the site of nerve injury were affected minimally $(\mathrm{GM} / \mathrm{crush} 7.67 \pm 0.33 \mathrm{mV}$, $\mathrm{GM} /$ cut $7.34 \pm 0.25 \mathrm{mV}$ ). Evoked EMG responses in musculature after sham surgery similarly revealed a transient subacute reduction in EMG amplitude immediately after surgery $(\mathrm{GM} /$ sham $7.81 \pm 0.41 \mathrm{mV}, \mathrm{TA} /$ sham $7.88 \pm 0.76$ $\mathrm{mV}$, GS/sham $6.87 \pm 0.71 \mathrm{mV}$, and PL/sham $6.06 \pm 0.65$ $\mathrm{mV})$. Reductions in EMG amplitude after sham surgery were observed to resolve within 2 weeks of surgery.

Serial assessment further elucidated various time courses of functional motor recovery after nerve injury with or without therapeutic electrical stimulation. EMG responses in musculature proximal to the nerve injury revealed no change in EMG amplitude, similar to that found after sham surgery $(\mathrm{GM} / \mathrm{sham} 9.33 \pm 0.49 \mathrm{mV}, \mathrm{GM} /$ crush $8.32 \pm 0.35 \mathrm{mV}, \mathrm{GM} /$ crush+stim $8.62 \pm 0.50 \mathrm{mV}, \mathrm{GM} / \mathrm{cut}$ $8.45 \pm 0.49 \mathrm{mV}$, and $\mathrm{GM} /$ cut+stim $8.80 \pm 0.49 \mathrm{mV}$ ) (Fig. 4). EMG recordings in GM muscle revealed preservation of $82.5 \%-98.2 \%$ of native function throughout the study irrespective of nerve injury or therapeutic modality (Table 1). EMG recordings in proximal musculature revealed a negligible effect of brief electrical stimulation on healthy muscle tissue unaffected by nerve injury. EMG recording in proximal musculature further confirmed the ability of implanted wireless stimulators to maximally recruit innervated musculature throughout the duration of the study.

\section{Serial Assessment of Nerve and Muscle Function After Nerve Crush Injury}

EMG recordings in TA and GS muscles revealed progressive functional recovery over 6 weeks after surgery. TA and GS muscles recovered $67.5 \%$ and $75.5 \%$ of native function 4 weeks after surgery, respectively, whereas PL muscles demonstrated recovery of only $49.2 \%$ of native function 4 weeks after surgery (Figs. 5-7). Serial EMG measurements revealed a reduced rate of functional recovery in the distal PL muscle compared with that of the more proximal TA and GS muscles. Thirteen weeks after surgery, TA, GS, and PL muscles demonstrated good functional recovery of motor function by regaining $96.4 \%$, $91.9 \%$, and $91.8 \%$ of native function, respectively.

In the presence of brief electrical stimulation, EMG responses in musculature distal to the site of nerve crush injury were increased significantly 2-4 weeks after surgery. EMG responses in TA and GS muscles 2 and 3 weeks after surgery in the presence of therapeutic electrical stimulation were significantly greater than in the absence of electrical stimulation (TA/crush $4.32 \pm 0.66 \mathrm{mV}$, TA/ crush+stim $6.01 \pm 0.59 \mathrm{mV}[\mathrm{p}=0.002] ;$ GS/crush $4.99 \pm$ $0.67 \mathrm{mV}, \mathrm{GS} /$ crush+stim $6.87 \pm 0.58 \mathrm{mV}[\mathrm{p}=0.002]$ ) (Figs. 5 and 6). EMG responses in PL muscles were significantly greater 2,3, and 4 weeks after surgery in the presence of therapeutic electrical stimulation than in its absence (PL/

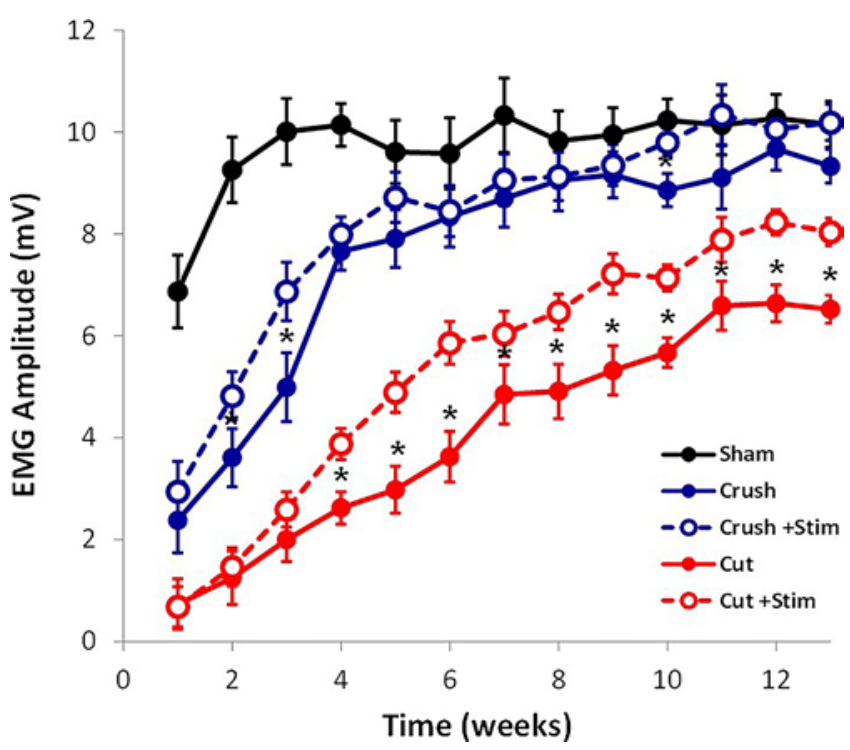

FIG. 6. Maximum amplitude of electromyograms evoked in the GS after electrical activation of uninjured, crushed, and cut-and-repaired sciatic nerves in both the presence and the absence of brief electrical stimulation via the implanted wireless nerve stimulator. Mean values and SDs are shown. ${ }^{*} p<0.05$ versus time-matched injury model with no electrical stimulation. Figure is available in color online only.

crush $3.64 \pm 0.34 \mathrm{mV}, \mathrm{PL} /$ crush+stim $5.78 \pm 0.35 \mathrm{mV}$ [p = 0.0005]) (Fig. 7). EMG recordings in TA, GS, and PL muscles 4 weeks after surgery revealed recovery of, respectively, $75.9 \%, 78.8 \%$, and $78.1 \%$ of native function in the presence of therapeutic electrical stimulation and $67.5 \%$, $75.5 \%$, and $49.2 \%$ of native function in the absence of therapeutic electrical stimulation (Table 1). At the terminal time point, TA, GS, and PL muscles all showed nearcomplete recovery of motor function in both the presence and the absence of therapeutic electrical stimulation.

\section{Serial Assessment of Nerve and Muscle Function After Nerve Transection and Repair}

EMG responses after transection injury revealed gradual progressive recovery of function over the 13-week postoperative period without electrical stimulation. EMG recordings in TA and GS muscles revealed recovery of, respectively, $25.1 \%$ and $25.8 \%$ of native function 4 weeks after surgery, whereas PL muscles demonstrated recovery of only $20.4 \%$. TA, GS, and PL muscles all demonstrated persistent motor deficits at the terminal time point, recovering only $65.4 \%, 64.2 \%$, and $66.8 \%$ of native function, respectively. Serial EMG assessment revealed variable rates of functional recovery in independent muscle groups, which highlights the delay in functional recovery experienced by distal PL muscles compared to that by the more proximal TA and GS muscles.

EMG responses in musculature distal to the site of nerve transection and repair were increased significantly between 4 and 13 weeks after surgery in the presence of brief electrical stimulation compared to musculature absent brief electrical stimulation. EMG responses in TA and PL muscles between 3 and 13 weeks after surgery were significantly greater in the presence of therapeutic elec- 


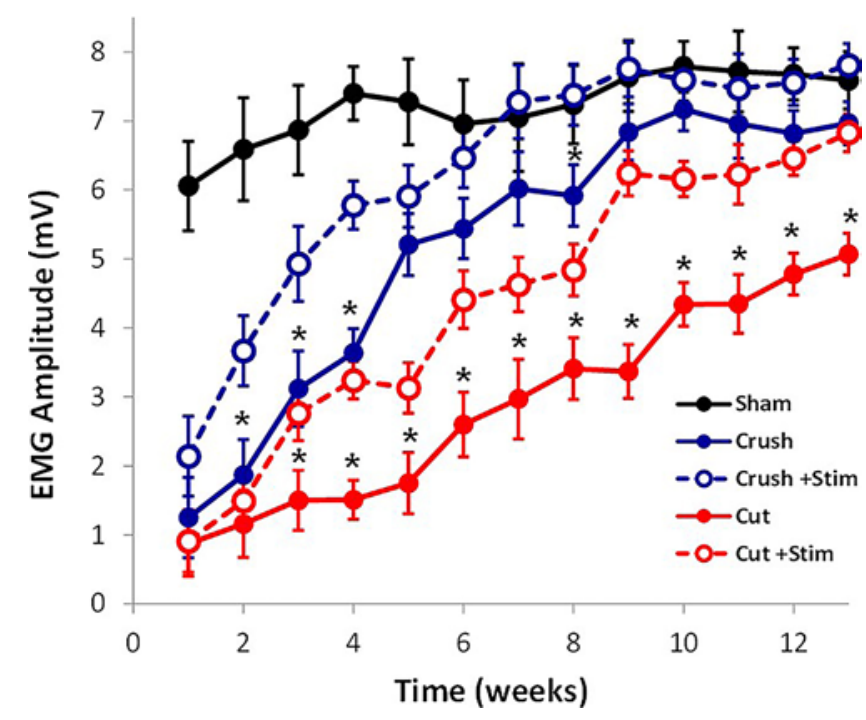

FIG. 7. Maximum amplitude of electromyograms evoked in the PL after electrical activation of uninjured, crushed, and cut-and-repaired sciatic nerves in both the presence and the absence of brief electrical stimulation via the implanted wireless nerve stimulator. Mean values and SDs are shown. ${ }^{*} p<0.05$ versus time-matched injury model with no electrical stimulation. From Ray et al: An update on addressing important peripheral nerve problems: challenges and potential solutions. Acta Neurochir (Wien) 159:1765-1773, 2017. Published with permission. Figure is available in color online only.

trical stimulation than in its absence (TA/cut $1.5 \pm 0.49$ $\mathrm{mV}$, TA/cut+stim $2.77 \pm 0.48 \mathrm{mV}[\mathrm{p}=0.01] ; \mathrm{PL} /$ cut 1.5 $\pm 0.44 \mathrm{mV}$, PL/cut+stim $2.76 \pm 0.39 \mathrm{mV}$ [p = 0.01]) (Figs. 5 and 7). EMG responses in GS muscles between 4 and 13 weeks after surgery were significantly greater in the presence of therapeutic electrical stimulation than in its absence (GS/cut $3.63 \pm 0.50 \mathrm{mV}$, GS/cut+stim $5.86 \pm 0.42$ $\mathrm{mV}[\mathrm{p}=0.0001]$ ) (Fig. 6). EMG recordings in TA, GS, and PL muscles 4 weeks after surgery revealed recovery of, respectively, $35.4 \%, 38.3 \%$, and $43.8 \%$ of native function in the presence of therapeutic electrical stimulation and $25.1 \%, 25.8 \%$, and $20.4 \%$ in its absence (Table 1). At the 13 -week postsurgery terminal time point, TA, GS, and PL muscles demonstrated recovery of, respectively, $79.0 \%$, $79.2 \%$, and $90.0 \%$ of native function in the presence of therapeutic electrical stimulation and $65.4 \%, 64.2 \%$, and $66.8 \%$ in its absence.

\section{Terminal Assessment of Nerve and Muscle Function}

Measurements of evoked muscle force production and wet muscle mass were made. Behavioral observations of progressive motor functional recovery after induced nerve injury were consistent with those found in EMG recordings, yet verification of terminal EMG measurements via gold-standard neuromuscular methodologies was desired.

As shown in Fig. 8 at the terminal time point for crush injury, TA and EDL muscles with and those without therapeutic electrical stimulation elicited maximal tetanic force measurements equivalent to those of TA and EDL muscles distal to uninjured nerve (TA/sham $5.2 \pm 1.0 \mathrm{~N}$, TA/crush $5.2 \pm 1.5 \mathrm{~N}, \mathrm{TA} /$ crush+stim $5.6 \pm 1.5 \mathrm{~N}, \mathrm{EDL} / \mathrm{sham} 3.2 \pm$ $0.3 \mathrm{~N}$, EDL/crush $3.3 \pm 0.9 \mathrm{~N}$, and EDL/crush+stim $3.4 \pm$
TABLE 1. Overview of functional recovery observed in rat musculature over various postoperative recovery times after nerve crush or transection injuries in the presence or absence of brief electrical stimulation

\begin{tabular}{clrrrr}
\hline \multirow{2}{*}{ Muscle } & & \multicolumn{4}{c}{ Postop Week } \\
\cline { 3 - 6 } GM & Injury Type & 1 & 4 & 8 & 13 \\
\hline & Crush & 98.2 & 89.8 & 82.5 & 89.3 \\
\hline & Crush+stim & 96.5 & 94.9 & 88.1 & 93.3 \\
\hline & Transection & 94.0 & 91.6 & 87.4 & 86.5 \\
\hline \multirow{2}{*}{ GS } & Transection+stim & 98.0 & 91.1 & 96.0 & 95.7 \\
\hline & Crush & 34.6 & 75.5 & 92.1 & 91.9 \\
\hline & Crush+stim & 42.8 & 78.8 & 92.9 & 100.4 \\
\hline \multirow{2}{*}{ TA } & Transection & 10.6 & 25.8 & 49.9 & 64.2 \\
\hline & Transection+stim & 9.9 & 38.3 & 65.8 & 79.2 \\
\hline & Crush & 19.3 & 67.5 & 89.8 & 96.4 \\
\hline & Crush+stim & 31.1 & 75.9 & 88.3 & 100.3 \\
\hline & Transection & 11.1 & 25.1 & 47.6 & 65.4 \\
\hline PL & Transection+stim & 11.5 & 35.4 & 64.2 & 79.0 \\
\hline & Crush & 20.6 & 49.2 & 81.8 & 91.8 \\
\hline & Crush+stim & 35.3 & 78.1 & 101.9 & 102.9 \\
\hline & Transection & 14.4 & 20.4 & 47.1 & 66.8 \\
\hline & Transection+stim & 15.0 & 43.8 & 66.9 & 90.0 \\
\hline
\end{tabular}

Values are presented as percentage of recovery compared to that seen in rats that underwent sham surgery, as measured by maximal EMG amplitudes obtained at the corresponding time points.

$0.7 \mathrm{~N}$ ). Maximum twitch force measurements in TA and EDL muscles distal to the site of nerve crush injury with and in those without therapeutic electrical stimulation revealed similar trends (TA/sham $2.2 \pm 0.4 \mathrm{~N}$, TA/crush 2.0 $\pm 0.2 \mathrm{~N}, \mathrm{TA} /$ crush+stim $2.2 \pm 0.5 \mathrm{~N}$, EDL/sham $1.2 \pm 0.2$ $\mathrm{N}$, EDL/crush $1.3 \pm 0.2 \mathrm{~N}$, and EDL/crush+stim $1.2 \pm 0.2$ $\mathrm{N})$. No difference was observed between maximal tetanic force measurements obtained from TA and EDL muscles in the presence or the absence of therapeutic electrical stimulation.

In contrast, TA and EDL muscles distal to the site of nerve transection injury with and those without therapeutic electrical stimulation elicited lower maximal tetanic force measurements than did TA and EDL muscles distal to uninjured nerve (TA/sham $5.2 \pm 1.0 \mathrm{~N}$, TA/cut $4.7 \pm 0.7$ $\mathrm{N}, \mathrm{TA} /$ cut+stim $4.8 \pm 0.9 \mathrm{~N}, \mathrm{EDL} /$ sham $3.2 \pm 0.3 \mathrm{~N}, \mathrm{EDL} /$ cut $0.7 \pm 0.2 \mathrm{~N}$, and EDL/cut+stim $1.2 \pm 0.2 \mathrm{~N}$ ). Maximal twitch force measurements revealed a similar trend. Interesting to note is that EDL muscles had significantly higher maximal tetanic force measurements in the presence of therapeutic electrical stimulation than in its absence (EDL/cut $0.7 \pm 0.2 \mathrm{~N}$, EDL/cut+stim $1.2 \pm 0.2 \mathrm{~N}$ [p $=0.031])$.

Measurements of wet muscle mass revealed trends similar to those observed with force measurements. TA and EDL muscles distal to the site of nerve crush injury with and those without therapeutic electrical stimulation possessed masses similar to those of TA and EDL muscles distal to uninjured nerve (TA/sham $1.10 \pm 0.20 \mathrm{~g}$, TA/ crush $1.05 \pm 0.13 \mathrm{~N}, \mathrm{TA} / \mathrm{crush}+\mathrm{stim} 1.06 \pm 0.15 \mathrm{~N}, \mathrm{EDL} /$ sham $0.37 \pm 0.06 \mathrm{~N}$, EDL/crush $0.28 \pm 0.03 \mathrm{~N}$, and EDL/ 

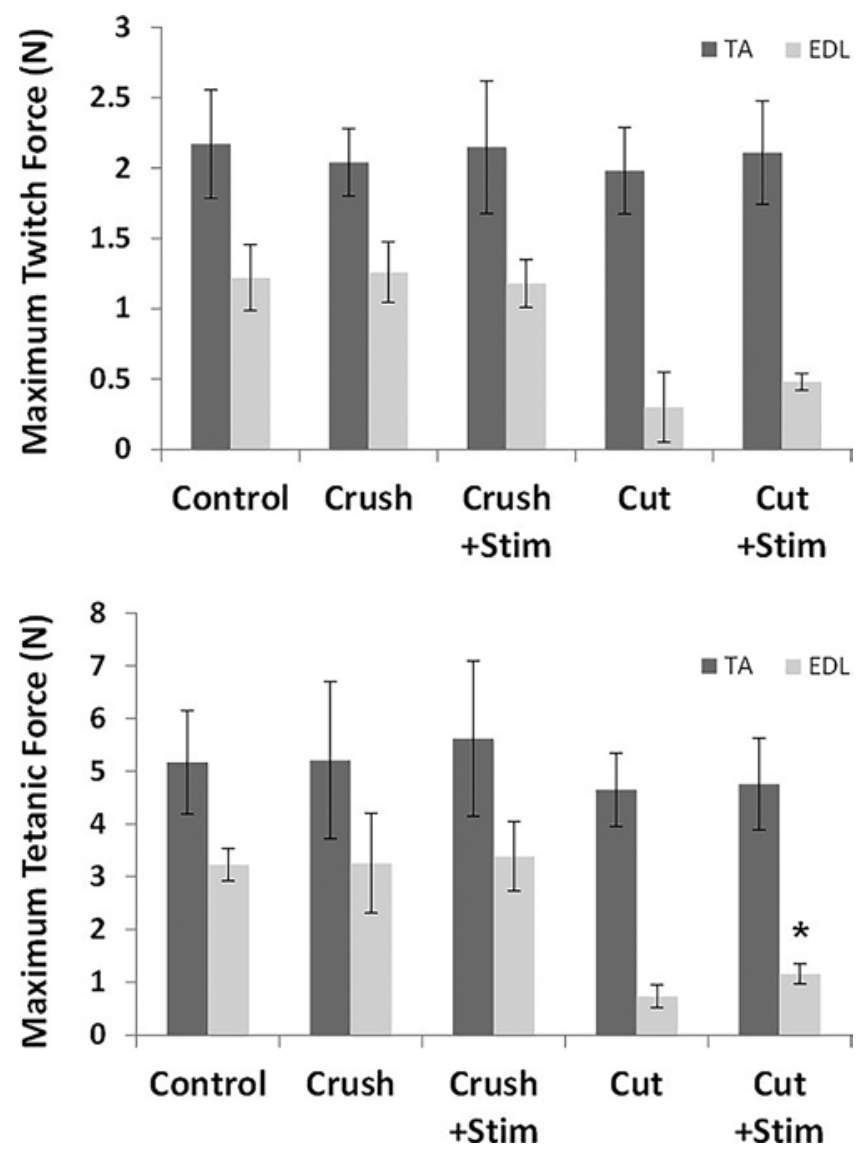

FIG. 8. Maximum isometric twitch forces (upper) and maximum isometric tetanic forces (lower) evoked by TA and EDL muscles after stimulation of uninjured, crushed, and cut-and-repaired sciatic nerves in both the presence and the absence of brief electrical stimulation via the implanted wireless nerve stimulator. Mean values and SDs are shown. ${ }^{*} p<$ 0.05 versus time-matched injury model with no electrical stimulation.

crush+stim $0.31 \pm 0.06 \mathrm{~N}$ ) (Fig. 9). TA and EDL muscles distal to the site of nerve transection injury had lower masses than TA and EDL muscles distal to uninjured nerve (TA/sham $1.10 \pm 0.20 \mathrm{~g}$, TA/cut $0.88 \pm 0.18 \mathrm{~N}$, TA/ cut+stim $0.94 \pm 0.15 \mathrm{~N}, \mathrm{EDL} / \mathrm{sham} 0.37 \pm 0.06 \mathrm{~N}, \mathrm{EDL} /$ cut $0.16 \pm 0.03 \mathrm{~N}$, and EDL/cut+stim $0.16 \pm 0.02 \mathrm{~N})$. TA muscles distal to the site of nerve transection injury had higher terminal muscle mass in the presence of therapeutic electrical stimulation than in its absence, although no significant differences were observed.

\section{Discussion}

Therapeutic electrical stimulation represents a nonpharmacologic adjunct for potentially improving peripheral nerve regeneration. Previous studies conducted by Gordon and coworkers $\mathrm{s}^{4,5,18}$ found that brief electrical stimulation of damaged peripheral nerve tissue can induce proregenerative phenotypes and accelerate functional recovery in vivo. Brief electrical stimulation of injured axons has been shown to increase cyclic AMP and activate protein kinase $\mathrm{A}$ in neuronal cell bodies and thereby enhance axonal outgrowth. ${ }^{19}$ Therapeutic electrical stimulation has been shown also to enhance nerve regeneration and functional

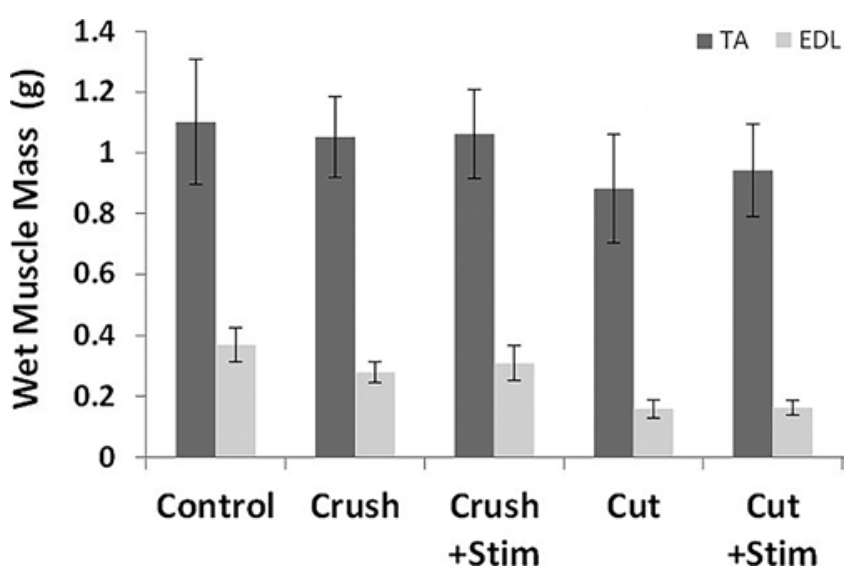

FIG. 9. Wet mass of TA and EDL muscles after sham surgery (no nerve injury), crush injury of the sciatic nerve, and cut-and-repair injury of the sciatic nerve in both the presence and the absence of brief electrical stimulation. Mean values and SDs are shown. ${ }^{*} p<0.05$ versus timematched injury model with no electrical stimulation.

recovery in rodent and human models. ${ }^{4,5,18}$ Yet, current techniques for applying therapeutic electrical stimulation are limited by the use of nerve hook or wire electrodes applied directly to the target nerve during open surgical repair of the injured nerve.

This report provides the first description of a novel implantable wireless nerve stimulator that enables successful delivery of therapeutic electrical stimulation to injured nerve tissue as a means of accelerating functional recovery in vivo. Brief electrical stimulation was applied to peripheral nerve tissue via a wireless implant after nerve crush and nerve transection injuries. These wireless nerve stimulators then were used to facilitate serial assessment of neuromuscular function after surgery in individual animals. Therapeutic electrical stimulation after nerve crush and nerve transection injuries was shown to accelerate functional recovery in multiple muscles below the level of injury and improve terminal motor recovery after nerve transection and repair. Altogether, these results suggest that this wireless implant system provides a unique and effective method of delivering therapeutic electrical stimulation to injured peripheral nerve tissue and a clinically relevant means of accelerating functional nerve regeneration after nerve injury.

In the absence of therapeutic electrical stimulation, functional recovery after nerve crush injury was observed over 6-8 weeks after surgery, and complete functional recovery was seen by 13 weeks. The rates of functional recovery observed in the absence of electrical stimulation matched those reported in previous studies, wherein complete functional recovery was observed 4-8 weeks after nerve crush injury. ${ }^{43}$ In contrast, the application of brief electrical stimulation directly after nerve crush injury was found to significantly increase functional recovery over 2-4 weeks after surgery. The increased rate of functional recovery observed in animals after nerve crush injury and brief electrical stimulation is consistent with that in previous reports that documented the positive effect of brief electrical stimulation after nerve crush and nerve compression injuries. ${ }^{18}$ It is important to note that although 
brief electrical stimulation accelerated acute functional recovery 2-4 weeks after nerve crush injury, therapeutic stimulation did not affect terminal functional recovery 13 weeks after surgery. Near-complete recovery of EMG and muscle force production in all animals after nerve crush injury provide additional evidence that brief electrical stimulation serves to accelerate the rate of functional nerve regeneration rather than to encourage supraphysiological innervation or activation of distal musculature.

Brief electrical stimulation applied via an implanted wireless nerve stimulator was observed also to accelerate functional recovery after nerve transection and repair. The use of wireless implants to track functional recovery in rodent nerve transection-and-repair models revealed various rates of recovery across motor targets in the presence and in the absence of brief electrical stimulation. In the absence of therapeutic electrical stimulation, progressive functional recovery was observed during the 13-week postsurgery recovery period. Persistent functional deficits observed at the terminal time point ranged from $40 \%$ to $60 \%$ maximal force production and were consistent with results of previous studies in which deficits were attributed to residual denervated muscle fibers..$^{22,40,43}$ However, the application of brief electrical stimulation after nerve transection significantly increased functional recovery after 4 weeks postsurgery. Improvements in functional recovery observed after brief electrical stimulation applied via the wireless nerve stimulator were similar to those achieved in previous studies that used conventional nerve hook electrodes and microwires. ${ }^{18}$ These results suggest that delivery of therapeutic electrical stimulation via a wireless nerve stimulator and proven methods of direct electrical stimulation are equally effective.

Unlike the acute transient response observed after nerve crush injury, brief electrical stimulation after nerve transection injury resulted in a long-lasting proregenerative effect that subsisted for the full length of the study. These results suggest that the positive therapeutic effect of brief electrical stimulation can last well beyond the initial dosing, a point highlighted in previous animal and human model study reports. ${ }^{4,5,18}$ The long-term effect of brief electrical stimulation was observed to be more prominent in musculature distal to the site of nerve injury (PL muscle) than in musculature proximal to the site of nerve injury (TA and GS muscles). While confirming subtle differences in the time courses of functional recovery related to the distance of axonal outgrowth, these results provide further evidence that electrical stimulation can accelerate the rate of axonal regeneration and the speed of muscle reinnervation.

Aside from effectively delivering therapeutic electrical stimulation, this system of thin-film wireless implants offers multiple advantages over existing methods of peripheral nerve interfacing. Because of the low profile and implantable nature of the device, our system can be simply deployed either at the time of peripheral nerve repair or at any time after surgery. After initial implantation, the wireless system provides a simple means of interfacing target nerves with optimal specificity and temporal control without requiring repeat placement of transcutaneous leads. Improved efficiency and specificity might ultimately result in improved clinical translation or future optimization of clinical treatment of peripheral nerve injuries. Future studies should examine the effect of brief electrical stimulation on the number and quality of regenerated axons as evaluated via histopathology and the optimal timing and duration of electrical stimulation after nerve injury.

\section{Conclusions}

The results of this study highlight the ability of a novel thin-film wireless implant to successfully deliver therapeutic electrical stimulation to injured peripheral nerve tissue and accelerate functional recovery. Compared with transcutaneous or percutaneous approaches, wireless nerve stimulators offer a completely implantable solution devoid of connecting wires and provide a lower risk of chronic infection. The absence of a solid-state power supply on the implantable stimulator increases the range of clinical applications by reducing the overall profile of the device. The passive nature of the implantable stimulator also offers greater safety and biocompatibility than battery-powered implants. Flexible and repeatable interfacing of targeted nerve tissue by an implanted wireless device might also facilitate new paradigms of therapeutic electrical stimulation that extend beyond the operating room. The use of implantable wireless stimulators might yield opportunities for repeat dosing of therapeutic electrical stimulation during postoperative recovery. Although yet unexplored, such techniques might facilitate improved clinical outcomes beyond those outlined in previous studies and leverage direct intraoperative nerve stimulation. Our wireless implant system might be ideally suited for eventual clinical translation and use in a variety of nerve-interfacing scenarios. We expect that ongoing work will further improve the current design and result in a clinically relevant platform that can be translated into the clinic.

\section{Acknowledgments}

This study was generously funded by the Department of Neurosurgery at Washington University in St. Louis, Missouri.

\section{References}

1. Ahlborn P, Schachner M, Irintchev A: One hour electrical stimulation accelerates functional recovery after femoral nerve repair. Exp Neurol 208:137-144, 2007

2. Al-Majed AA, Neumann CM, Brushart TM, Gordon T: Brief electrical stimulation promotes the speed and accuracy of motor axonal regeneration. J Neurosci 20:2602-2608, 2000

3. Brenner MJ, Moradzadeh A, Myckatyn TM, Tung TH, Men$\operatorname{dez} \mathrm{AB}$, Hunter DA, et al: Role of timing in assessment of nerve regeneration. Microsurgery 28:265-272, 2008

4. Brushart TM, Hoffman PN, Royall RM, Murinson BB, Witzel C, Gordon T: Electrical stimulation promotes motoneuron regeneration without increasing its speed or conditioning the neuron. J Neurosci 22:6631-6638, 2002

5. Brushart TM, Jari R, Verge V, Rohde C, Gordon T: Electrical stimulation restores the specificity of sensory axon regeneration. Exp Neurol 194:221-229, 2005

6. Campbell WW: Evaluation and management of peripheral nerve injury. Clin Neurophysiol 119:1951-1965, 2008

7. Dagum AB: Peripheral nerve regeneration, repair, and grafting. J Hand Ther 11:111-117, 1998

8. Dahlin LB: The role of timing in nerve reconstruction. Int Rev Neurobiol 109:151-164, 2013 
9. Dahlin LB: Techniques of peripheral nerve repair. Scand J Surg 97:310-316, 2008

10. Dillon GP, Yu X, Bellamkonda RV: The polarity and magnitude of ambient charge influences three-dimensional neurite extension from DRGs. J Biomed Mater Res 51:510-519, 2000

11. English AW, Schwartz G, Meador W, Sabatier MJ, Mulligan A: Electrical stimulation promotes peripheral axon regeneration by enhanced neuronal neurotrophin signaling. Dev Neurobiol 67:158-172, 2007

12. Evans GR: Peripheral nerve injury: a review and approach to tissue engineered constructs. Anat Rec 263:396-404, 2001

13. Evans PJ, Mackinnon SE, Best TJ, Wade JA, Awerbuck DC, Makino AP, et al: Regeneration across preserved peripheral nerve grafts. Muscle Nerve 18:1128-1138, 1995

14. Fox IK, Mackinnon SE: Experience with nerve allograft transplantation. Semin Plast Surg 21:242-249, 2007

15. Gamble P, Stephen M, MacEwan M, Ray WZ: Serial assessment of functional recovery following nerve injury using implantable thin-film wireless nerve stimulators. Muscle Nerve 54:1114-1119, 2016

16. Gans C: Fiber architecture and muscle function. Exerc Sport Sci Rev 10:160-207, 1982

17. Geremia NM, Gordon T, Brushart TM, Al-Majed AA, Verge VMK: Electrical stimulation promotes sensory neuron regeneration and growth-associated gene expression. Exp Neurol 205:347-359, 2007

18. Gordon T, Amirjani N, Edwards DC, Chan KM: Brief postsurgical electrical stimulation accelerates axon regeneration and muscle reinnervation without affecting the functional measures in carpal tunnel syndrome patients. Exp Neurol 223:192-202, 2010

19. Gordon T, Udina E, Verge VM, de Chaves EI: Brief electrical stimulation accelerates axon regeneration in the peripheral nervous system and promotes sensory axon regeneration in the central nervous system. Mot Contr 13:412-441, 2009

20. Hudson TW, Liu SY, Schmidt CE: Engineering an improved acellular nerve graft via optimized chemical processing. Tissue Eng 10:1346-1358, 2004

21. Jesuraj NJ, Santosa KB, Macewan MR, Moore AM, Kasukurthi R, Ray WZ, et al: Schwann cells seeded in acellular nerve grafts improve functional recovery. Muscle Nerve 49:267-276, 2014

22. Kalliainen LK, Jejurikar SS, Liang LW, Urbanchek MG, Kuzon WM Jr: A specific force deficit exists in skeletal muscle after partial denervation. Muscle Nerve 25:31-38, 2002

23. Kline DG: Physiological and clinical factors contributing to the timing of nerve repair. Clin Neurosurg 24:425-455, 1977

24. Kline DG: Surgical repair of peripheral nerve injury. Muscle Nerve 13:843-852, 1990

25. Lee JY, Giusti G, Wang H, Friedrich PF, Bishop AT, Shin AY: Functional evaluation in the rat sciatic nerve defect model: a comparison of the sciatic functional index, ankle angles, and isometric tetanic force. Plast Reconstr Surg 132:1173-1180, 2013

26. Mackinnon SE, Doolabh VB, Novak CB, Trulock EP: Clinical outcome following nerve allograft transplantation. Plast Reconstr Surg 107:1419-1429, 2001

27. McCaig CD: Nerve branching is induced and oriented by a small applied electric field. J Cell Sci 95:605-615, 1990

28. McCaig CD: Nerve growth in the absence of growth cone filopodia and the effects of a small applied electric field. J Cell Sci 93:715-721, 1989

29. McCaig CD, Rajnicek AM: Electrical fields, nerve growth and nerve regeneration. Exp Physiol 76:473-494, 1991

30. Méndez J, Keys A: Density and composition of mammalian muscle. Metabolism 9:184-188, 1960
31. Mendonça AC, Barbieri CH, Mazzer N: Directly applied low intensity direct electric current enhances peripheral nerve regeneration in rats. J Neurosci Methods 129:183-190, 2003

32. Moore AM, MacEwan M, Santosa KB, Chenard KE, Ray WZ, Hunter DA, et al: Acellular nerve allografts in peripheral nerve regeneration: a comparative study. Muscle Nerve 44:221-234, 2011

33. Myckatyn TM, Mackinnon SE: A review of research endeavors to optimize peripheral nerve reconstruction. Neurol Res 26:124-138, 2004

34. Navarro X, Vivó M, Valero-Cabré A: Neural plasticity after peripheral nerve injury and regeneration. Prog Neurobiol 82:163-201, 2007

35. Noble J, Munro CA, Prasad VS, Midha R: Analysis of upper and lower extremity peripheral nerve injuries in a population of patients with multiple injuries. J Trauma 45:116-122, 1998

36. Politis MJ, Zanakis MF, Albala BJ: Facilitated regeneration in the rat peripheral nervous system using applied electric fields. J Trauma 28:1375-1381, 1988

37. Román GC, Strahlendorf HK, Coates PW, Rowley BA: Stimulation of sciatic nerve regeneration in the adult rat by low-intensity electric current. Exp Neurol 98:222-232, 1987

38. Song B, Zhao M, Forrester J, McCaig C: Nerve regeneration and wound healing are stimulated and directed by an endogenous electrical field in vivo. J Cell Sci 117:4681-4690, 2004

39. Swett JE, Wikholm RP, Blanks RH, Swett AL, Conley LC: Motoneurons of the rat sciatic nerve. Exp Neurol 93:227252, 1986

40. Urbanchek MG, Picken EB, Kalliainen LK, Kuzon WM Jr: Specific force deficit in skeletal muscles of old rats is partially explained by the existence of denervated muscle fibers. J Gerontol A Biol Sci Med Sci 56:B191-B197, 2001

41. Urbanchek MS, Chung KC, Asato H, Washington LN, Kuzon WM Jr: Rat walking tracks do not reflect maximal muscle force capacity. J Reconstr Microsurg 15:143-149, 1999

42. Varejão AS, Melo-Pinto P, Meek MF, Filipe VM, Bulas-Cruz $\mathrm{J}$ : Methods for the experimental functional assessment of rat sciatic nerve regeneration. Neurol Res 26:186-194, 2004

43. Wood MD, Kemp SW, Weber C, Borschel GH, Gordon T: Outcome measures of peripheral nerve regeneration. Ann Anat 193:321-333, 2011

44. Xie J, MacEwan MR, Liu W, Jesuraj N, Li X, Hunter D, et al: Nerve guidance conduits based on double-layered scaffolds of electrospun nanofibers for repairing the peripheral nervous system. ACS Appl Mater Interfaces 6:9472-9480, 2014

45. Yan Y, Sun HH, Mackinnon SE, Johnson PJ: Evaluation of peripheral nerve regeneration via in vivo serial transcutaneous imaging using transgenic Thy1-YFP mice. Exp Neurol 232:7-14, 2011

\section{Disclosures}

W.Z.R. has served as a consultant for Globus and has ownership in Acera Surgical, Inc.

\section{Author Contributions}

Conception and design: Ray. Acquisition of data: MacEwan, Gamble, Stephen. Analysis and interpretation of data: Stephen. Drafting the article: MacEwan. Critically revising the article: Ray, MacEwan. Reviewed submitted version of manuscript: all authors. Statistical analysis: MacEwan. Administrative/technical/material support: Stephen. Study supervision: Ray.

\section{Correspondence}

Wilson Z. Ray: Washington University School of Medicine, St. Louis, MO.rayz@wudosis.wustl.edu. 